\title{
Fungistatic Action of Juglans
}

\author{
SHAHNAZ AHMAD, MUKHTAR A. WAHID, AND A. Q. S. BUKHARI \\ Karachi Laboratories, Pakistan Council of Scientific and Industrial Research, Karachi-\$9, Pakistan
}

Received for publication 26 July 1972

A crude extract of the bark of Juglans regia Linn, tested against 13 species of fungi, showed selective fungistatic action against some species of the dermatophytes tested.

Juglans regia Linn (Juglandaceae), commonly known as the walnut, is a large, deciduous tree abundantly found in Baluchistan, North Iran, the Caucasus, Armenia, and other temperate regions. Its bark is said to prevent the formation of tartar of gums (1). It is also reported to be anthelmintic, antidysenteric, and detergent (1, 7 ), and Wren (8) has reported that walnut is used in cases of herpes, eczema, scrofula, and ulcers. Bactericidal properties of the Juglans were reported by Herrman (3) and Largange (4). It is also said to be useful for the treatment of fungal infections, including ringworm infections of the skin (2). However, there has been no systematic investigation of the fungistatic properties of the walnut.

Since the incidence and recurrence of fungal infections is great in the temperate regions, and in view of the reported utility of this bark in the treatment of certain skin infections, we decided to assess its in vitro fungistatic potency before undertaking any clinical trials with Juglans.

Butylaminohydroxybenzoate (Jadit) is claimed to be an effective antiparasitic and antimycotic agent. Rosenkraenzer (6) reported that $96.3 \%$ of patients suffering from fungal infections were cured with butylaminohydroxybenzoate. Jadit (batch no. HU) was obtained from a local chemist and has been selected for comparative reference.

The bark of $J$. regia was obtained from a local market. It was ground to coarse fibers, $1 \mathrm{~kg}$ of which was soaked in alcohol $(2 \times 10$ liters $)$ and shaken on an automatic shaker for $72 \mathrm{~h}$. The alcohol was evaporated at $45 \mathrm{C}$ under reduced pressure $(17.5 \mathrm{~cm} \mathrm{of} \mathrm{Hg})$ to give a crude alcoholic extract.

A water-soluble extract was prepared by dissolving this residue in $150 \mathrm{ml}$ of petroleum ether and shaking it with $250 \mathrm{ml}$ of water in a separating funnel. The aqueous portion was then removed for subsequent reevaporation in vacuo.

Trichophyton rubrum, T. violaceum, T. gourvila, $T$. schoenleini, Microsporum gypseum, M. andouini, $M$. vanbrenseghemii, Sporotrichum schenckii, Epidermophyton flocosum, Candida albicans, C. tropicalis, Aspergillus, Fusarium, and Helminthosporium species were selected for testing.

Fungistatic action was assessed by the method of Reddish (5).

Melted sterile Sabouraud dextrose-agar was poured into sterile petri dishes and allowed to solidify. The surface was then swabbed with 96h-old standing cultures of these fungi. Cavities were made in the centers of the petri dishes by a sterile cork borer (1.3-cm diameter), and the cavities were filled with $0.3 \mathrm{ml}$ of 1,2 , and $5 \%$ alcoholic extracts and aqueous extracts of $J$. regia. All the petri dishes were prepared in triplicate and incubated at $37 \mathrm{C}$. The control plates carried the vehicle and were run concurrently. The zones of inhibition were measured after $96 \mathrm{~h}$ in each case. The experiment described in Table 1 was repeated six times, and the experiment described in Table 2 was repeated three times.

The study on $J$. regia indicates that its bark has promising fungistatic qualities. An alcoholic extract of the bark of $J$. regia Linn tested against 13 available species of the fungi (Table 1) was effective against dermatophytes, which are well known for causing superficial infections of the keratinized areas of the body, i.e., skin, hair, and nails. The extract showed maximal activity against Microsporum gypseum and M. audouini, whereas Microsporum vanbreuseghemii remained unaffected (Table 1). This extract is also effective against the Trichophyton, Sporotrichum, and Candida species, but the fungistatic activity is comparatively lower than that exhibited against $M$. gypseum and M. audouini, and it is absolutely inactive against the so-called nonpathogenic fungal species, Aspergillus, Fusarium, and Helminthosporium. In fact, $A$. niger grows abundantly on the surface of a solution of Juglans (Bukhari and Ahmad, unpublished data). It has a selective fungistatic action against some species of these dermatophytes, which seems to be proportional to the concentration of the drug, although this activity appears insignificant only when the mean inhibitions of 
TABLE 1. Fungistatic action of an alcoholic extract of Juglans regia

\begin{tabular}{|c|c|c|c|}
\hline \multirow{2}{*}{ Specimen of fungus ${ }^{a}$} & \multicolumn{3}{|c|}{ Zones of inhibition (cm) } \\
\hline & $1 \%$ Alcohol & 2\% Alcohol & 5\% Alcohol \\
\hline 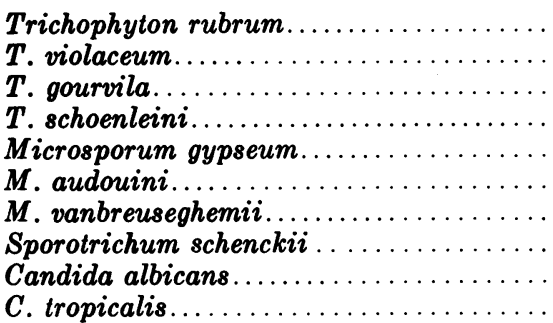 & $\begin{array}{l}3.6 \pm 0.07 \\
3.4 \pm 0.06 \\
3.5 \pm 0.06 \\
3.4 \pm 0.05 \\
3.9 \pm 0.03 \\
3.9 \pm 0.09 \\
\pm=0 \\
3.4 \pm 0.05 \\
2.4 \pm 0.04 \\
2.6 \pm 0.09\end{array}$ & $\begin{array}{l}3.8 \pm 0.05 \\
3.7 \pm 0.06 \\
3.5 \pm 0.06 \\
3.7 \pm 0.05 \\
4.1 \pm 0.08 \\
4.2 \pm 0.06 \\
3.7 \pm 0.06 \\
2.7 \pm 0.07 \\
2.9 \pm 0.05\end{array}$ & $\begin{array}{l}4.7 \pm 0.04 \\
4.8 \pm 0.05 \\
4.8 \pm 0.06 \\
4.6 \pm 0.03 \\
5.0 \pm 0.06 \\
5.2 \pm 0.06 \\
4.2 \pm 0.05 \\
3.6 \pm 0.1 \\
3.6 \pm 0.07\end{array}$ \\
\hline
\end{tabular}

a There was no activity against Aspergillus, Fusarium sp., or Helminthosporium sp.

b Mean plus or minus standard error.

c Dash (-) represents no activity.

TABLE 2. Comparative fungistatic action of Juglans and Jadita

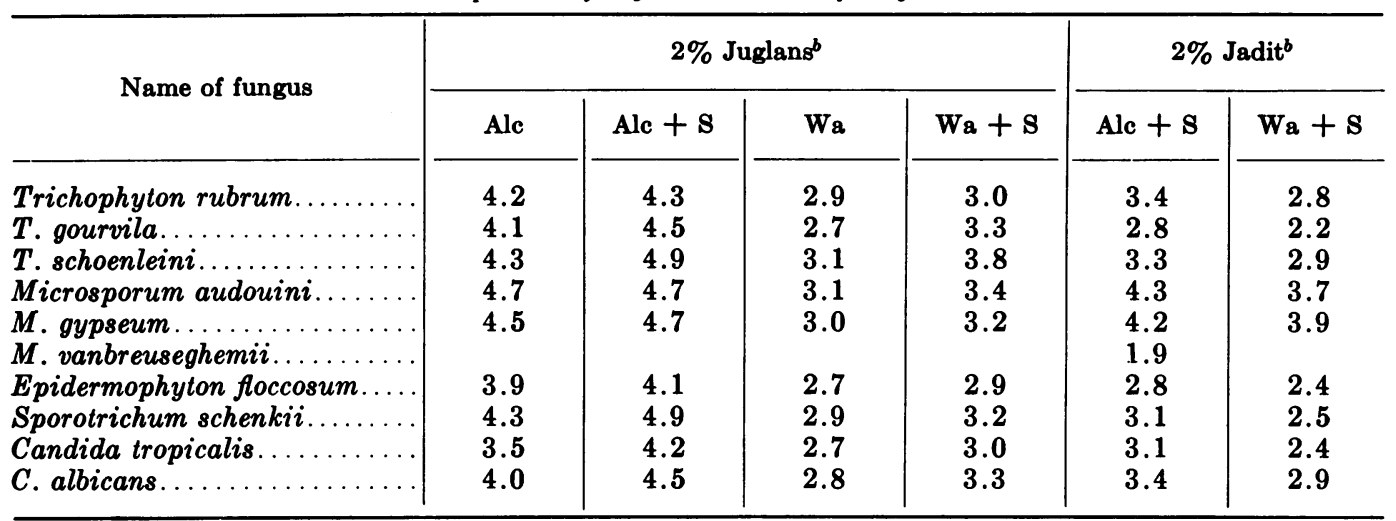

a Values represent the means of three experiments.

b Alc, Alcohol; + S, $1 \%$ salicylic acid; wa, water.

$1 \%$ are compared with those of a $2 \%$ solution (Table 1).

Comparative studies utilizing a $2 \%$ solution of Juglans and Jadit are summarized in Table 2, which shows that Juglans exhibits better fungistatic action against Trichophyton, Microsporum, Candida, and Epidermophyton species than does Jadit. The alcoholic solutions of both Juglans and Jadit are better than their water-soluble counterparts. It was interesting to record that a solution of Juglans prepared in alcohol exhibits significantly stronger fungistatic action than an equally potent solution prepared in water (Table 2), although alcohol as such has no effect on any of the fungi. The mechanism whereby the addition of alcohol enhances the activity of Juglans is not understood; it may be assumed that alcohol might potentiate the action of Juglans.
When compared with butylaminohydroxybenzoate, the well-known antimycotic agent, Juglans appears to exert greater in vitro inhibition of locally available species of fungi. Addition of $1 \%$ salicylic acid to Juglans does not significantly enhance the action of Juglans, which may be attributed to the fact that the studies were performed on fungi in vitro.

The present study relates to a crude extract of the $J$. regia Linn; therefore, any solution of Juglans thus prepared does not represent a true quantitative sample in contrast to that of butylaminohydroxybenzoate. Investigations are in progress to isolate the active principle from Juglans and assess it on dermatophytic infections of the skin.

We are grateful to Ahmed Kamal, T.Pk., the former Director, for extending facilities and encouragement for the completion of this work. We also thank Khursheed 
Khan, University of Karachi, for providing some of the fungal cultures, and Shamim Qureshi and Manzaruddin Alvi for their help in preparation of the extracts.

\section{LITERATURE CITED}

1. Chopra, R. N., I. C. Chopra, K. L. Handa, and L. D. Kapur. 1958. Indigenous drugs of India, 2nd ed. UN Dhur \& Sons, Private Ltd., Calcutta.

2. Dorlan, W. A. N. 1951. Quoted in American illustrated medical dictionary, 22nd ed. Saunders and Co., Philadelphia.

3. Herrman, G. 1956. Med. Mont. 10:790.
4. Largange, E. 1954. C. R. Acad. Sci. 50C. Biol. 148: 2097.

5. Reddish, G. F. 1957. Antiseptics, disinfectants, fungicides and chemical and physical sterilization. Lea \& Febiger, Philadelphia.

6. RosenKraenzer, R. 1959. Medizin Heute, 8 p. 257-59. Essen, W. Germany.

7. Watt, G. 1890 . A dictionary of the economic products of India, vol. IV. W. H. Allen \& Co., London.

8. Wren, R. C. 1956. Potters new cyclopedia of botanical drugs and preparations. Sir Isaac Pitman \& Sons Ltd., London. 